\title{
THE FORGOTTEN BRIDGE
}

A CASE REPORT

Caria T, Rodriques N, Mer S, Oliveira MI, Calhau R, Gonçalves N

Anesthesiology Department, Centro Hospitalar de Lisboa Ocidental - Portugal

Aspiration or dislodgement of an odontogenic foreign material may result in serious complications
including laceration, perforation and hemorrhage of the esophagus and gastrointestinal tract or
or even airway obstruction ${ }^{1}$.
The aim of this case report is to show that even an apparently uneventful general anesthesia, may
ultimately lead to unpleasant surprises.

43-year-old-woman

- Surgery: Elective hysteroscopy and polypectomy

- Medical History: Dyslipidemia + class II obesity

- Airway:

$\rightarrow$ No signs of predictable difficulties

$\rightarrow$ No loose teeth, crowns, or dentures.

- Anesthesia: General anesthesia (i-gel laryngeal mask nr4)

- Post-anesthetic recovery unit:

$\rightarrow$ Missing her fixed partial denture (bridge)

$\rightarrow$ No symptoms of airway or esophageal obstruction

$\rightarrow$ Chest radiography - dental prosthesis in the esophagus

- Endoscopy - denture removed uneventfully.

The presence of a dental prosthesis or loose tooth represents a threat to the patient's safety, especially if the airway is accessed ${ }^{2,3}$. Although most foreign bodies entering the oropharynx pass through the gastrointestinal tract without complications, it may be necessary to identify and remove it to avoid late complications ${ }^{1,3}$.

A preanesthetic evaluation, including oral examination, is essential to ensure patient safety ${ }^{1,2}$.

In our case, the assessment was performed but the patient omitted the presence of a bridge, putting herself into unnecessary risk.
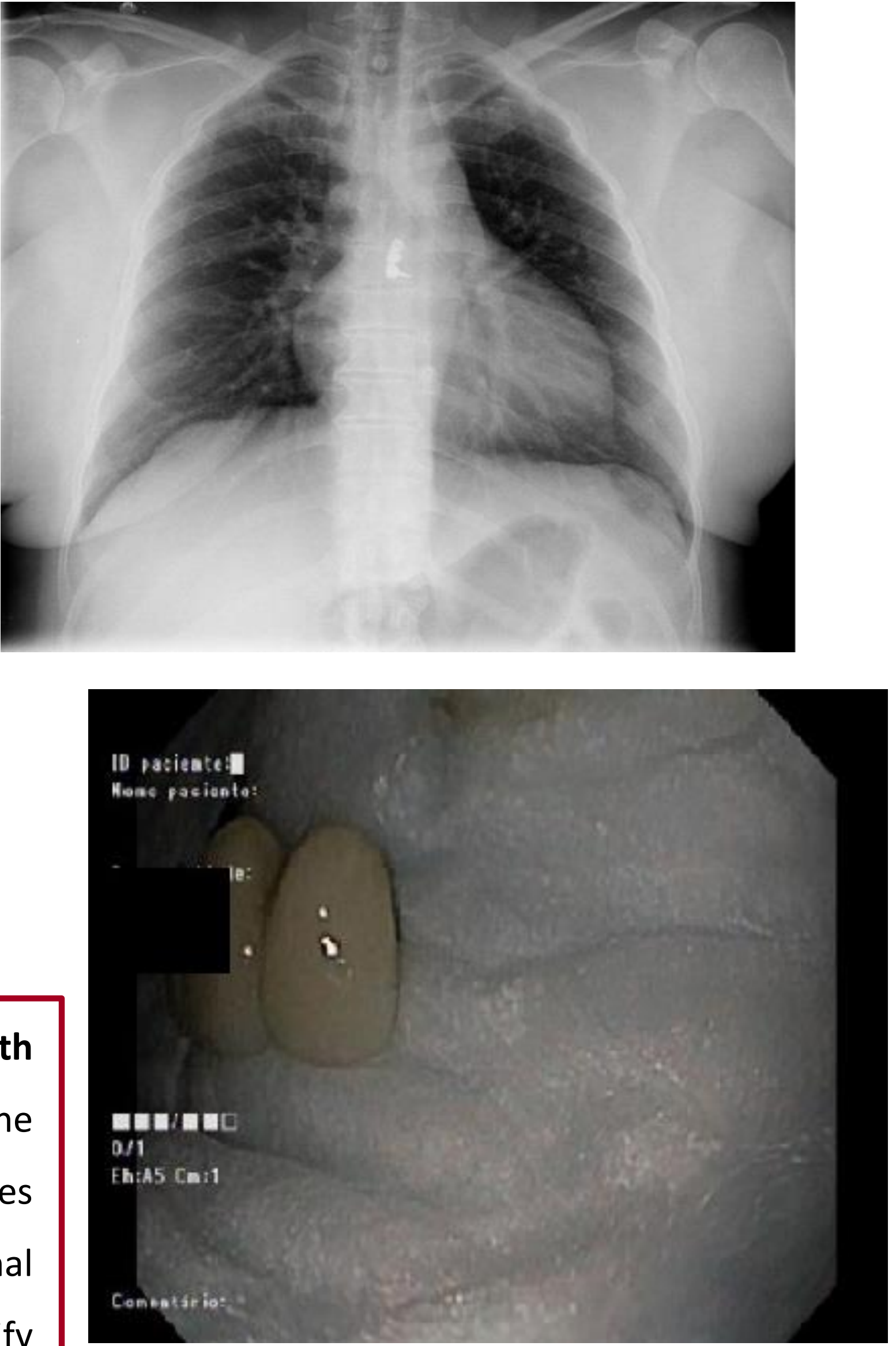

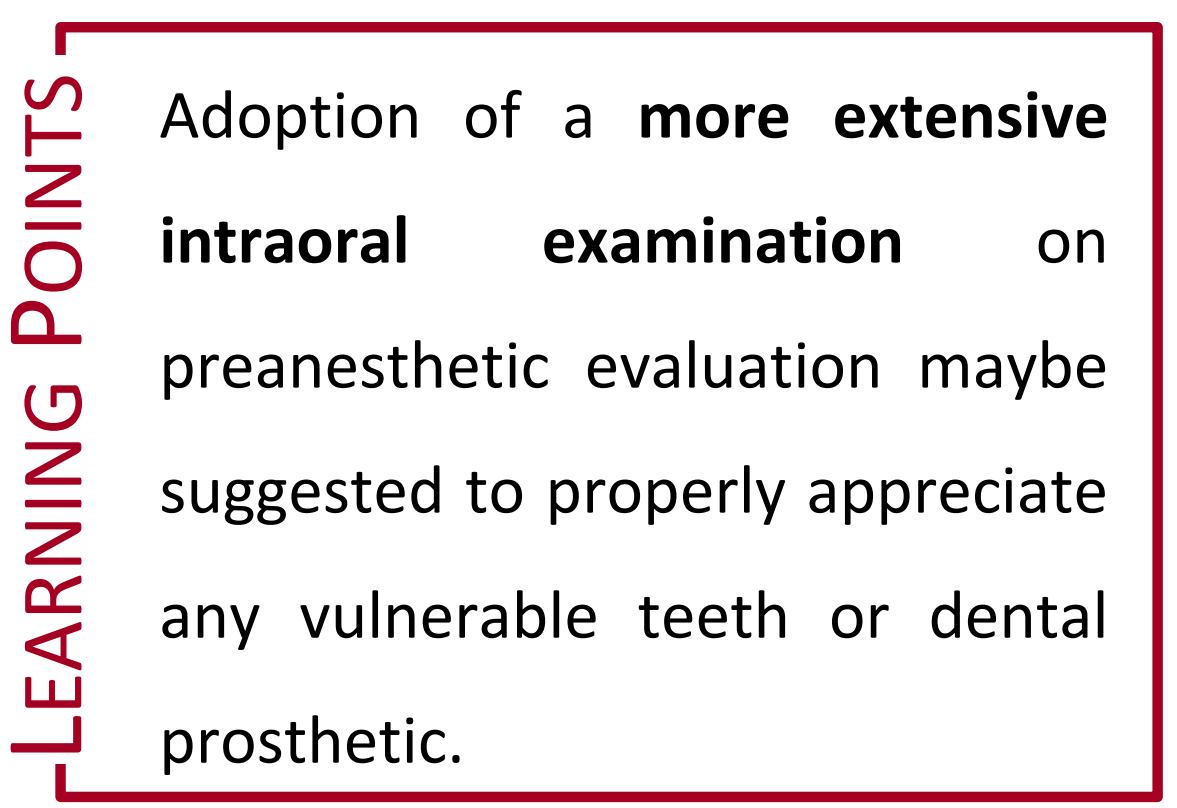

